\title{
Acute effects of feeding fructose, glucose and sucrose on blood lipid levels and systemic inflammation
}

\author{
Faizan Jameel ${ }^{1}$, Melinda Phang ${ }^{1,3}$, Lisa G Wood ${ }^{1,2}$ and Manohar L Garg ${ }^{1 *}$
}

\begin{abstract}
Background: Recent studies have demonstrated a relationship between fructose consumption and risk of developing metabolic syndrome. Mechanisms by which dietary fructose mediates metabolic changes are poorly understood. This study compared the effects of fructose, glucose and sucrose consumption on post-postprandial lipemia and low grade inflammation measured as hs-CRP.

Methods: This was a randomized, single blinded, cross-over trial involving healthy subjects $(n=14)$. After an overnight fast, participants were given one of 3 different isocaloric drinks, containing $50 \mathrm{~g}$ of either fructose or glucose or sucrose dissolved in water. Blood samples were collected at baseline, 30, 60 and 120 minutes post intervention for the analysis of blood lipids, glucose, insulin and high sensitivity C-reactive protein (hs-CRP).

Results: Glucose and sucrose supplementation initially resulted in a significant increase in glucose and insulin levels compared to fructose supplementation and returned to near baseline values within 2 hours. Change in plasma cholesterol, LDL and HDL-cholesterol (measured as area under curve, AUC) was significantly higher when participants consumed fructose compared with glucose or sucrose $(P<0.05)$. AUC for plasma triglyceride levels however remained unchanged regardless of the dietary intervention. Change in AUC for hs-CRP was also significantly higher in subjects consuming fructose compared with those consuming glucose $(P<0.05)$, but not sucrose $(P=0.07)$.

Conclusion: This study demonstrates that fructose as a sole source of energy modulates plasma lipids and hsCRP levels in healthy individuals. The significance of increase in HDL-cholesterol with a concurrent increase in LDL-cholesterol and elevated hs-CRP levels remains to be delineated when considering health effects of feeding fructose-rich diets.
\end{abstract}

Registration number for clinical trials: ACTRN12614000431628

\section{Introduction}

Fructose, commonly known as fruit sugar, is also a major component of sweeteners such as table sugar, honey and high fructose corn syrup (HFCS). Fructose intake has quadrupled since the beginning of 20th century, partly because of the introduction of HFCS [1]. Increased fructose consumption can lead to a parallel rise in cardiovascular disease risk factors, i.e. increase in blood lipids [2,3], development of insulin resistance [4,5], alteration in the production of satiety hormones (insulin, leptin

\footnotetext{
* Correspondence: manohar.garg@newcastle.edu.au

'Nutraceuticals Research Group, School of Biomedical Sciences \& Pharmacy, University of Newcastle, 305C Medical Science Building, Callaghan, NSW 2308, Australia

Full list of author information is available at the end of the article
}

and ghrelin) [3], increase in inflammatory biomarkers $[6,7]$ and increase in obesity $[5,8]$. Since similar effects do not occur following the intake of starch or glucose, it has been proposed that fructose-induced metabolic changes are not mediated by excessive sugar intake in general, but are specific to fructose. Precise underlying mechanisms by which fructose consumption may induce negative metabolic effects are not clear. One recent study demonstrated that in young healthy individuals, consumption of glucose and fructose drinks resulted in markedly different hemodynamic responses, with fructose stimulating a sustained increase in blood pressure [9]. These observations support the concept that diets 
with repeated fructose loads may, over time, contribute to increased cardiovascular disease risk.

The aim of this study was to investigate the effects of fructose compared to glucose and sucrose consumption, on postprandial lipemia and low grade inflammation in healthy subjects. Previous studies have examined the effects of feeding sugars as part of a meal on cardiovascular disease indices. In the current study, we looked at postprandial lipid and low grade inflammation following a single dose of sugary drink given as a sole source of energy after an overnight fast.

\section{Methods}

\section{Study population}

Healthy male and female adults $(\mathrm{n}=14)$ between the ages of 18-60 years were recruited by advertisement and underwent study procedures at the Nutraceuticals Research Group Clinic rooms, University of Newcastle, NSW, Australia. Exclusion criteria were: diagnosed hyperlipidaemia, diabetes, gastrointestinal disorders, currently on fructose/sugar restricted diet, vegan diet or weight loss program, undergone any surgical procedure for obesity, pregnant or lactating mother, taking lipid-lowering or anti-inflammatory drugs and BMI $>30 \mathrm{~kg} / \mathrm{m}^{2}$. Participants were asked to complete a medical questionnaire, International Physical Activity Questionnaire (IPAQ) [10] and a $24 \mathrm{hr}$ food record. Approval for the study was granted by the Human Research Ethics Committee of the University of Newcastle, Australia. All participants provided written informed consent and the study was conducted in accordance with The Declaration of Helsinki. The trial was registered with the Australian \& New Zealand Clinical Trials Registry (ACTRN12614000431628). Body composition was assessed by bioimpedance analysis (BIA) using single frequency bioelectrical impedance apparatus (Maltron International, Essex, UK). Measurements were conducted in the supine position, with participants wearing light clothing and without shoes, in the morning after a minimum 10 hour fast. Participants were asked to refrain from physical exertion and alcohol consumption for 24 hours prior to testing.

\section{Study design}

The trial was a randomised, single blinded, controlled cross-over intervention trial. Participants visited the research facility on three separate occasions, where they consumed one of 3 different isocaloric sugary drinks on each occasion, with a minimum of one week wash out period in between. The participants were randomised to consume: 1) $50 \mathrm{~g}$ fructose dissolved in $300 \mathrm{ml}$ of water 2) $50 \mathrm{~g}$ glucose dissolved in $300 \mathrm{ml}$ of water and 3) $50 \mathrm{~g}$ sucrose dissolved in $300 \mathrm{ml}$ of water. Each sugar drink contained $10 \mathrm{ml}$ of lemon juice to provide a more uniform and palatable taste. Participants were asked to consume the sweetened drinks within 2-3 minutes and compliance was observed. Block randomization technique was used for allocation of participants to treatment arms. During each visit, a fasting blood sample was collected prior to supplementation, then 30,60 and 120 minutes following intake of the sugary drink. The participants remained in the research facility until the final sample was collected and were asked to limit physical activity during their time in the research facility.

\section{Laboratory methods}

Blood samples at base line, 30, 60 and 120 minutes were collected into tubes pre-coated with EDTA, lithium heparin and sodium fluoride by venepuncture. EDTA blood tubes were centrifuged for 10 minutes at $3000 \mathrm{~g}$ at $4^{\circ} \mathrm{C}$ for separation of plasma and stored at $-80^{\circ} \mathrm{C}$ for further use. The lithium heparin tubes for blood lipids and sodium fluoride tubes for blood glucose and insulin measurement were analysed by Hunter New England Area Pathology Services (Newcastle). Twenty four hour food recalls were collected by face to face interview by an inhouse dietician. Food records collected from participants were entered into FoodWorks Version 7.0.291 database (Xyris Software Pty Ltd, Queensland, Australia) to analyse daily energy and nutrient intake of participants.

\section{Statistical analysis}

All data are presented as mean $\pm \mathrm{SEM}$. Area under the curve (AUC) was calculated by the trapezoid method. Preliminary assumption testing was done to check for normality, linearity, outliers and homogeneity of variance with no serious violations noted for all test variables within the three different groups of fructose, glucose and sucrose. Comparisons between different groups were made with one-way repeated measures ANOVA and post hoc Tukey testing. A probability level of $\mathrm{p}<0.05$ was adopted throughout to determine statistical significance unless otherwise mentioned. All statistical analyses were carried out with SPSS software (version 21.0; SPSS Inc., Chicago, IL, USA).

\section{Results}

All participants were healthy and their baseline values of anthropometric measurements and blood biomarkers were within normal range (Table 1).

As shown in Figure 1A, at 30 minutes, fructose consumption was followed by an increase in blood glucose levels that was significantly lower than following glucose and sucrose consumption. At 60 and 120 minutes there were no differences between the 3 groups. The overall change in blood glucose levels followed by fructose consumption measured as area under the curve (AUC), was significantly lower than following glucose supplementation (Figure 1B). 
Table 1 Baseline values of anthropometric measurements, blood biomarkers \& energy and nutrients intakes of study participants

\begin{tabular}{|c|c|c|c|}
\hline \multicolumn{4}{|c|}{ A. Baseline characteristics } \\
\hline & Total & Males & Females \\
\hline $\mathrm{n}$ & 14 & 07 & 07 \\
\hline Age (Yrs) & $28.0 \pm 0.79$ & $28.7 \pm 0.64$ & $27.3 \pm 1.45$ \\
\hline Weight (kg) & $69.6 \pm 3.48$ & $78.8 \pm 4.26$ & $60.4 \pm 2.50$ \\
\hline BMI $\left(\mathrm{kg} / \mathrm{m}^{2}\right)$ & $24.2 \pm 0.72$ & $25.6 \pm 0.82$ & $22.8 \pm 0.98$ \\
\hline SMM (kg) & $30.8 \pm 2.44$ & $34.1 \pm 2.72$ & $27.4 \pm 3.83$ \\
\hline FFM (kg) & $51.1 \pm 3.48$ & $60.0 \pm 4.44$ & $42.1 \pm 2.49$ \\
\hline PBF (\%) & $27.1 \pm 1.88$ & $24.0 \pm 1.86$ & $30.1 \pm 2.96$ \\
\hline Waist: hip & $0.8 \pm 0.00$ & $0.9 \pm 0.00$ & $0.8 \pm 0.12$ \\
\hline Glucose (mmol/L) & $4.7 \pm 0.12$ & $4.7 \pm 0.19$ & $4.6 \pm 0.16$ \\
\hline Cholesterol (mmol/L) & $4.2 \pm 0.22$ & $4.3 \pm 0.37$ & $4.1 \pm 0.25$ \\
\hline Triglyceride (mmol/L) & $1.01 \pm 0.20$ & $1.35 \pm 0.35$ & $0.67 \pm 0.11$ \\
\hline LDL-C (mmol/L) & $2.43 \pm 0.22$ & $2.72 \pm 0.39$ & $2.14 \pm 0.19$ \\
\hline $\mathrm{HDL}-\mathrm{C}(\mathrm{mmol} / \mathrm{L})$ & $1.32 \pm 0.13$ & $1.00 \pm 0.09$ & $1.65 \pm 0.19$ \\
\hline Total/HDL ratio & $3.6 \pm 0.38$ & $4.5 \pm 0.52$ & $2.6 \pm 0.25$ \\
\hline Insulin (mIU/L) & $9.2 \pm 1.09$ & $9.5 \pm 1.56$ & $8.8 \pm 1.65$ \\
\hline $\mathrm{CRP}(\mathrm{mg} / \mathrm{L})$ & $1.5 \pm 0.31$ & $1.7 \pm 0.47$ & $1.5 \pm 0.45$ \\
\hline \multicolumn{4}{|c|}{ B: Daily energy and nutrient intake } \\
\hline Daily intake & Total & Males & Females \\
\hline Energy No DF (kj) & $8619 \pm 1001$ & $10077 \pm 1797$ & $7161 \pm 641$ \\
\hline Energy DF (kj) & $8820 \pm 1016$ & $10292 \pm 1828$ & $7348 \pm 645$ \\
\hline Protein (g) & $89.37 \pm 11.78$ & $107.82 \pm 21.00$ & $70.92 \pm 6.86$ \\
\hline Fat (g) & $73.29 \pm 10.20$ & $90.56 \pm 16.78$ & $56.03 \pm 8.99$ \\
\hline Cholesterol (mg) & $204.46 \pm 43.01$ & $227.38 \pm 81.43$ & $181.53 \pm 34.81$ \\
\hline Carbohydrate (g) & $481.54 \pm 224.56$ & $293.89 \pm 58.01$ & $669.18 \pm 451.01$ \\
\hline Sugar (g) & $88.21 \pm 12.24$ & $110.05 \pm 21.72$ & $76.20 \pm 11.41$ \\
\hline Glucose (g) & $10.40 \pm 2.32$ & $9.54 \pm 4.27$ & $11.27 \pm 2.19$ \\
\hline Sucrose (g) & $32.70 \pm 9.06$ & $35.50 \pm 17.15$ & $29.90 \pm 7.67$ \\
\hline Bound fructose $(\mathrm{g})$ & $16.35 \pm 4.53$ & $17.75 \pm 8.57$ & $14.95 \pm 3.83$ \\
\hline Free fructose (g) & $12.56 \pm 2.27$ & $11.60 \pm 3.93$ & $13.51 \pm 2.57$ \\
\hline Total fructose (g) & $28.91 \pm 6.51$ & $29.55 \pm 12.36$ & $28.46 \pm 5.56$ \\
\hline Lactose (g) & $12.36 \pm 2.09$ & $16.13 \pm 2.92$ & $8.60 \pm 2.40$ \\
\hline Maltose (g) & $18.01 \pm 5.84$ & $25.74 \pm 10.06$ & $10.29 \pm 5.15$ \\
\hline
\end{tabular}

Mean values \pm standard error of mean.

BMI, Body mass index; SMM, Skeletal muscle mass, FM, Fat Free Mass; PBF Percentage Body Fat; LDL-C, Low Density Lipoprotein cholesterol; HDL-C, High Density Lipoprotein cholesterol; CRP, c-Reactive Protein, DF: dietary fibre.

Figure $1 \mathrm{C}$ demonstrates that after 30 minutes, fructose consumption led to a smaller increase in insulin levels than glucose or sucrose. At 60 minutes, insulin levels in the fructose fed group remained lower than the glucose fed group. However, at 120 minutes, there were no differences in insulin levels across intervention groups. The overall increase in the insulin levels, measured as AUC, was significantly lower when participants consumed fructose compared to glucose or sucrose (Figure 1D).

Fructose consumption led to an increase in total cholesterol compared to glucose and sucrose consumption (Figure 2A). The effects were still apparent at 60 minutes, however at 120 minutes there were no differences between groups. The overall increase in plasma total cholesterol measured as AUC was significantly higher when participants consumed fructose compared to glucose or sucrose (Table 2). At 30 minutes after fructose consumption we observed an increase in LDL-cholesterol compared to glucose and sucrose (Figure 2B). The effects were still apparent at 60 minutes. However, at 120 minutes there were no differences between groups. Overall, the increase in LDL cholesterol measured as AUC was significantly higher when participants consumed fructose compared to glucose or sucrose (Table 2). Similarly, fructose consumption was followed by an increase in HDL-cholesterol at 30 minutes in comparison to glucose and sucrose (Figure 2C). The effects were still apparent at 60 minutes; however, at 120 minutes there were no differences between groups. The overall increase in HDL cholesterol measured as AUC was significantly higher when participants consumed fructose compared to glucose or sucrose (Table 2).

Comparison of all treatment groups revealed no significant difference in TG levels at 30 minutes. At $60 \mathrm{mi}-$ nutes, subjects fed fructose had lower TG levels compared to glucose. At 120 minutes there were no differences between groups (Figure 2D). There were overall no significant differences in plasma triglyceride levels (measured as AUC) regardless of the dietary intervention (Table 2). Comparison between all treatment groups revealed no significant change in the ratio of total/HDL-cholesterol at all the time points $(\mathrm{p}>0.005)$. Furthermore, the overall change in the ratio of total/ HDL-cholesterol measured as AUC was not different between groups (Table 2).

Figure $3 \mathrm{~A}$ demonstrates that fructose consumption was followed by an increase in hs-CRP level at $30 \mathrm{mi}$ nutes when compared to glucose and sucrose. At $60 \mathrm{mi}-$ nutes, hs-CRP was not different compared to glucose or sucrose and at 120 minutes there were no differences between groups. There was, however, an overall increase in hs-CRP levels measured as AUC in subjects who consumed fructose compared with those who consumed glucose $(\mathrm{p}<0.05)$, but not sucrose (Figure $3 \mathrm{~B})$.

\section{Discussion}

This study was designed to examine the metabolic consequences of sugar consumption when it is used as a sole source of energy. Participants consumed fructose in the fasting state in the morning; therefore, majority of the sugar consumed would be used to produce energy 


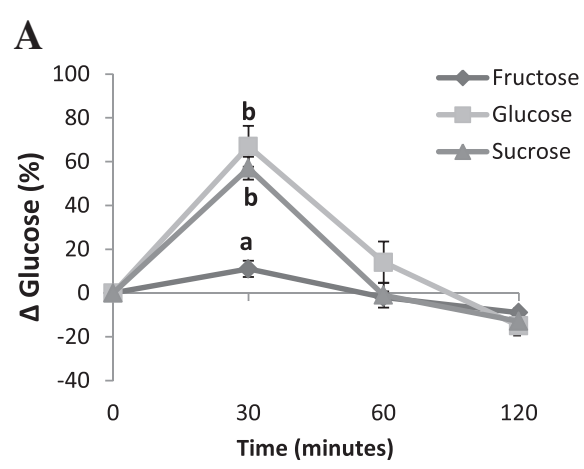

C

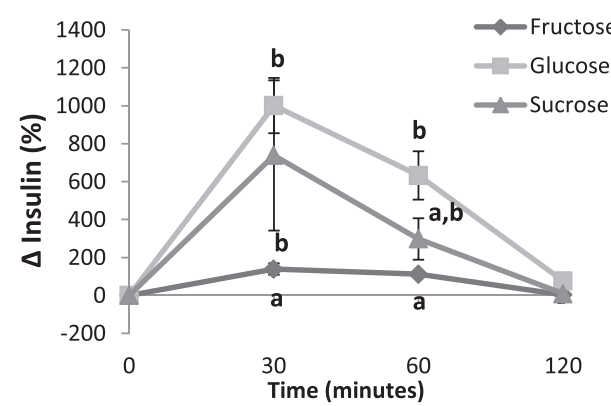

B

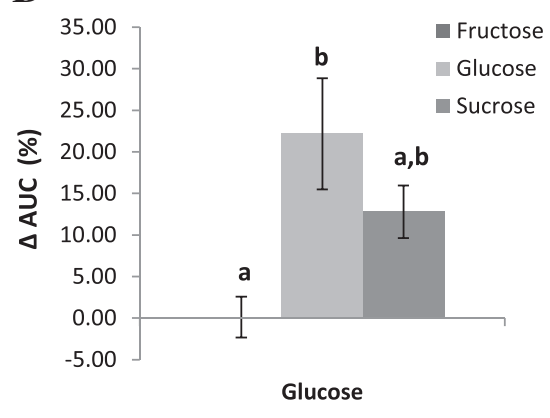

D

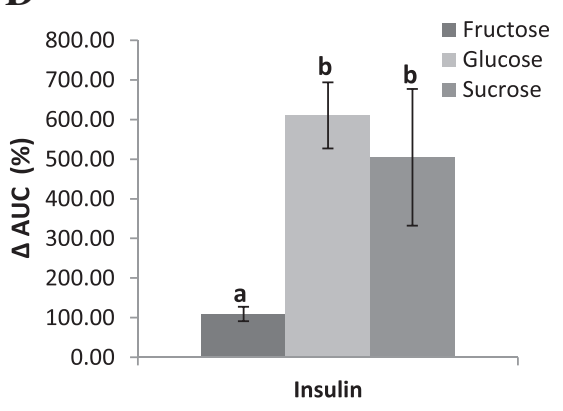

Figure 1 Kinetics of change and area under the curve (AUC) for blood glucose (mmol/L) (A and B respectively) and insulin (mIU/L) ( $C$ and $D$ respectively) after the fructose, glucose or sucrose supplementations. Values without a common superscript are significantly different; $P<0.05$.
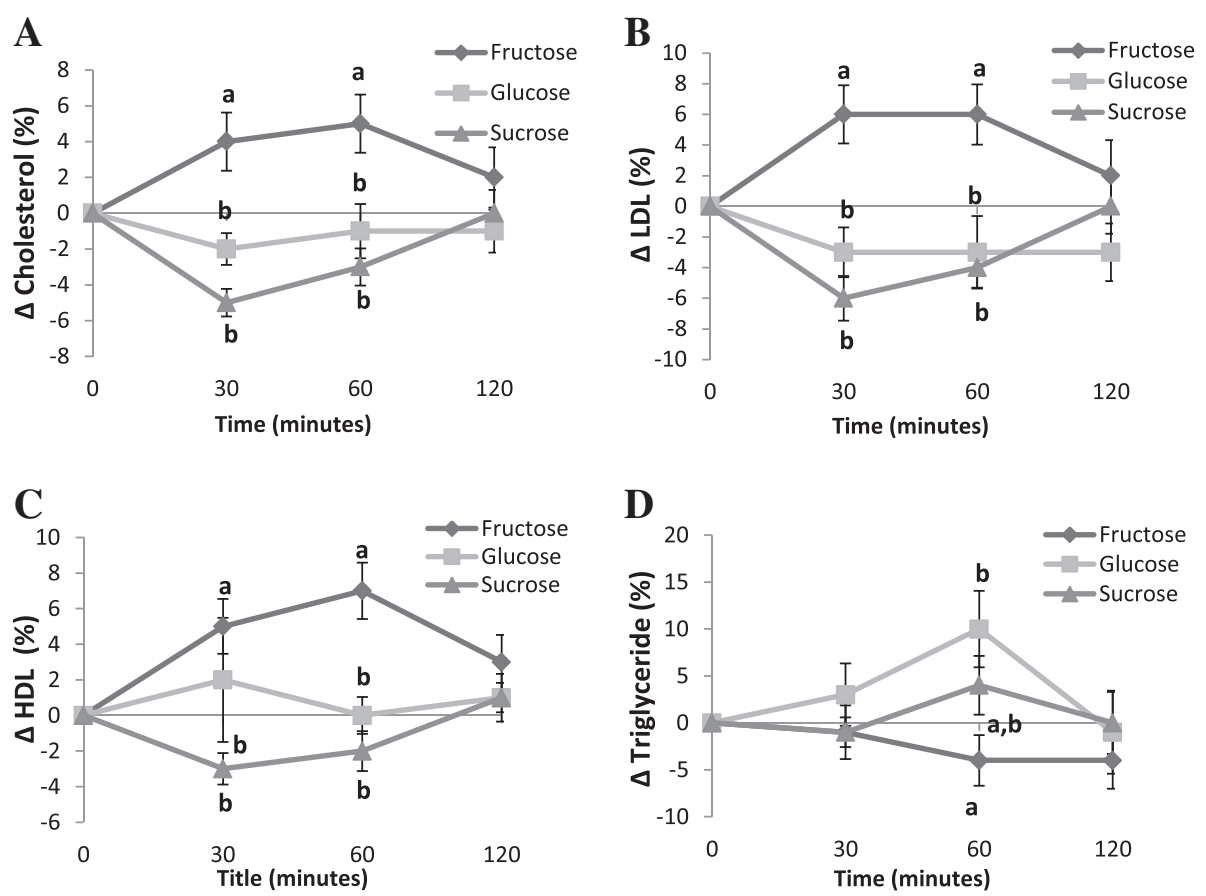

Figure 2 Kinetics of change for (A) total cholesterol (mmol/L); (B) LDL-C (mmol/L); (C)HDL-C (mmol/L) and (D) triglyceride (mmol/L) after the fructose, glucose or sucrose supplementations. Values without a common superscript are significantly different; $P<0.05$. 
Table 2 Area under curve (AUC) for blood lipids derived from kinetics of change

\begin{tabular}{lccc}
\hline \multicolumn{4}{l}{ Area under curve (AUC) } \\
\hline & Fructose (F) & Glucose (G) & Sucrose (S) \\
\hline Cholesterol (mmol/L) & $4.09 \pm 1.67^{\mathrm{a}}$ & $-0.90 \pm 1.25^{\mathrm{b}}$ & $-2.41 \pm 0.85^{\mathrm{b}}$ \\
$\mathrm{LDL}-\mathrm{C}(\mathrm{mmol} / \mathrm{L})$ & $4.50 \pm 2.03^{\mathrm{a}}$ & $-3.22 \pm 1.69^{\mathrm{b}}$ & $-3.87 \pm 1.25^{\mathrm{b}}$ \\
HDL-C $(\mathrm{mmol} / \mathrm{L})$ & $6.69 \pm 1.56^{\mathrm{a}}$ & $0.63 \pm 1.70^{\mathrm{b}}$ & $-0.84 \pm 0.60^{\mathrm{b}}$ \\
Triglyceride $(\mathrm{mmol} / \mathrm{L})$ & $-2.45 \pm 2.37$ & $5.65 \pm 3.52$ & $1.79 \pm 2.68$ \\
Total/HDL ratio & $-1.97 \pm 0.92$ & $-0.62 \pm 0.73$ & $-1.57 \pm 0.95$ \\
\hline
\end{tabular}

Mean values \pm standard error of mean; LDL-C, Low Density Lipoprotein cholesterol; HDL-C, High Density Lipoprotein cholesterol; Values without a common superscript are significantly different, $\mathrm{P}<0.05$.

and/or partly used to replenish glycogen stores at this time of day [11]. Fructose was a sole source of energy without accompanying meal and there was no other nutrient to augment postprandial lipemia. Consumption of sugar sweetened beverages in the morning in the fasting state separated the effect of fructose from excess energy intake which may be a confounder in the overfeeding studies [11]. This is the first study to report the consequences of consuming a beverage containing fructose, glucose or sucrose as a sole source of energy, on postprandial lipid levels and inflammation markers. Acute fructose consumption in a single dose of $50 \mathrm{~g} /$ day which provided approximately $8 \%$ of daily energy in the form of a beverage, resulted in a significant increase in the plasma levels of total, LDL and HDL cholesterol and the acute phase pro-inflammatory marker hs-CRP, compared to the same dose of glucose or sucrose. Interestingly, no significant change in TG levels was observed.

The change in fasting glucose and insulin responses was modest in fructose compared with glucose and sucrose groups. This modest increase in the glucose and insulin levels after fructose consumption is consistent with previous studies [12-14]. The blunted rise in insulin in response to fructose consumption is consistent with the blunted rise in blood glucose level, but may also be partly attributed to less release of intestinal incretin hormone that binds to $\beta$-cells of the pancreas leading to reduce secretion of insulin [15].

Changes in total, LDL and HDL-cholesterol levels were significantly higher when participants consumed fructose compared with glucose or sucrose sweetened beverage. Previous studies examining postprandial lipemia following fructose consumption were either focused on triglyceride levels only $[11,14,16-19]$ or demonstrate heterogeneous results. Two studies showed no effects of fructose on plasma total, LDL or HDL-cholesterol levels [20,21], while another 2 studies [2,22] showed a significant increase in fasting serum total and LDL cholesterol following 4-5 weeks of consuming fructose-rich diets compared to the starch diet. The reason for the increase in postprandial levels of total, LDL and HDL cholesterol in subjects who consumed fructose in our study is not known. Since no nutrients, other than sugars, were included in the test beverages, the lipoproteins measured were almost exclusively of hepatic origin. Whether fructose can influence total and LDL-cholesterol levels by blocking LDL-receptors or affect HDL cholesterol via CETP or reverse cholesterol transport is not known, therefore, merits further investigation.

Dietary fructose has been previously shown to cause either no change $[2,21,23,24]$ or an increase in fasting plasma triglycerides in healthy subjects $[20,25]$. Our results showed no significant change in postprandial triglyceride levels irrespective of the type of sugar. These findings are in agreement with a meta-analysis reporting no significant change in post-prandial TG level unless the amount of fructose exceeds $50 \mathrm{~g} /$ day [26]. On the other hand, the present results are in contrast with literature reporting an increase in TG after acute fructose consumption [3,11,14,16-18,25,27]. This disagreement may be due to the accompanying meal with consumption of the fructose beverage in previously published studies. In our study, due to the absence of other energy yielding nutrients, the clearance rate of triglycerides can be expected to be higher, resulting in overall no change. Moreover the shorter duration (2 hours) of our study compared to the study by Bohannan et al. [19] (5 hours)
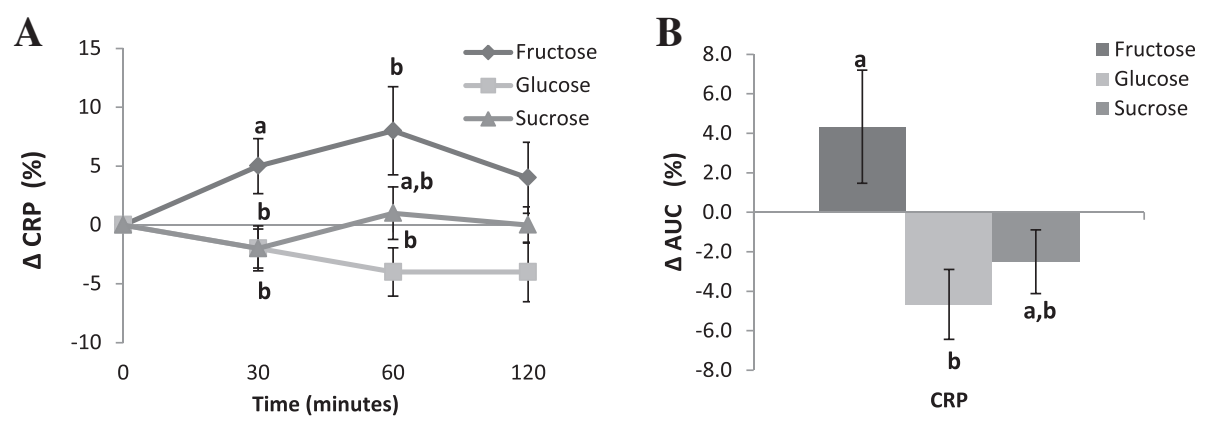

Figure 3 Kinetics of change (A) and area under the curve (AUC) (B) for CRP (mg/L) after the fructose, glucose or sucrose supplementations. Values without a common superscript are significantly different; $P<0.05$. 
may account for the discrepancy in the two studies. Therefore, the lipemic effects of fructose may depend on the dose and duration of fructose feeding and whether fructose is consumed in the presence or absence of other energy nutrients.

The pro-inflammatory biomarker that we examined in this study was hs-CRP. The area under the curve for hsCRP level was significantly increased in the fructose group compared with glucose, but not with sucrose. This is the first time, to our knowledge, in healthy and normal weight adult subjects, that acute fructose consumption has been shown to increase hs-CRP levels. Previous studies reporting an increase in hs-CRP level were either conducted in a mixed population of lean and overweight individuals [12] or a long term intervention study [6]. The proposed mechanism of fructose-induced oxidative stress and inflammation markers (TNF $\alpha$, IL-6, IL-1 $\beta$ ) [28] potentially resulting in an increase synthesis of hs-CRP merits further investigation. Fructose has been shown to induce oxidative stress in cellular [29] and animal models [30], thereby, may result in elevated levels of pro-inflammatory mediators. Whether fructose can directly (without conversion to fat) influence inflammation pathways (leukotriene synthesis, expression of adhesion molecules etc) remains to be delineated. In conclusion, this study demonstrates that fructose as a sole source of energy modulates plasma lipids and hsCRP levels in healthy individuals. However, the significance of increase in HDL-cholesterol with a concurrent increase in LDL-cholesterol and elevated hsCRP levels remains to be delineated when considering health effects of feeding fructose-rich diets.

\section{Competing interests}

The authors declare that they have no competing interests.

\section{Authors' contributions}

MLG \& LGW designed the project; MP provided technical assistance and help in data analysis; FJ conducted research, analyzed data, drafted the paper and had primary responsibility for final content. All authors read and approved the final manuscript.

\section{Acknowledgements}

This study was funded by a grant from the Hunter Medical Research Institute. The authors declare no conflict of interest.

\section{Author details \\ ${ }^{1}$ Nutraceuticals Research Group, School of Biomedical Sciences \& Pharmacy, University of Newcastle, 305C Medical Science Building, Callaghan, NSW 2308, Australia. ${ }^{2}$ Centre for Asthma and Respiratory Disease, School of Biomedical Sciences \& Pharmacy, University of Newcastle, Callaghan, NSW, Australia. 'Women's and Children's Health Research Institute, Women's and Children Hospital, North Adelaide, South Australia, Australia.}

Received: 29 August 2014 Accepted: 13 December 2014 Published: 16 December 2014

\section{References}

1. Semchyshyn HM: Fructation in vivo: detrimental and protective effects of fructose. Biomed Res Int 2013, 2013:9.

2. Swanson JE, Laine DC, Thomas W, Bantle JP: Metabolic effects of dietary fructose in healthy subjects. Am J Clin Nutr 1992, 55(4):851-856.
3. Teff KL, Elliott SS, Tschop M, Kieffer TJ, Rader D, Heiman M, Townsend RR, Keim NL, D'Alessio D, Havel PJ: Dietary fructose reduces circulating insulin and leptin, attenuates postprandial suppression of ghrelin, and increases triglycerides in women. J Clin Endocrinol Metab 2004, 89(6):2963-2972.

4. Le KA, Tappy L: Metabolic effects of fructose. Curr Opin Clin Nutr Metab Care 2006, 9(4):469-475.

5. Stanhope KL, Schwarz JM, Keim NL, Griffen SC, Bremer AA, Graham JL, Hatcher B, Cox CL, Dyachenko A, Zhang W, McGahan JP, Seibert A, Krauss RM, Chiu S, Schaefer EJ, Ai M, Otokozawa S, Nakajima K, Nakano T, Beysen C, Hellerstein MK, Berglund L, Havel PJ: Consuming fructose-sweetened, not glucosesweetened, beverages increases visceral adiposity and lipids and decreases insulin

sensitivity in overweight/obese humans. J Clin Investig 2009, 119(5):1322-1334.

6. Aeberli I, Gerber PA, Hochuli M, Kohler S, Haile SR, Gouni-Berthold I, Berthold HK, Spinas GA, Berneis K: Low to moderate sugar-sweetened beverage consumption impairs glucose and lipid metabolism and promotes inflammation in healthy young men: a randomized controlled trial. Am J Clin Nutr 2011, 94(2):479-485.

7. Cox CL, Stanhope KL, Schwarz JM, Graham JL, Hatcher B, Griffen SC, Bremer AA, Berglund L, McGahan JP, Keim NL, Havel PJ: Circulating concentrations of monocyte chemoattractant protein-1, plasminogen activator inhibitor-1, and soluble leukocyte

adhesion molecule-1 in overweight/obese men and women consuming fructose- or glucose-sweetened beverages for 10 weeks. J Clin Endocrinol Metab 2011, 96(12):E2034-E2038.

8. Tordoff MG, Alleva AM: Effect of drinking soda sweetened with aspartame or high-fructose corn syrup on food intake and body weight. Am J Clin Nutr 1990, 51(6):963-969.

9. Brown $C M$, Dulloo AG, Yepuri G, Montani JP: Fructose ingestion acutely elevates blood pressure in healthy young humans. Am J Physiol Regul Integr Comp Physiol 2008, 294(3):R730-R737.

10. Craig CL, Marshall AL, Sjostrom M, Bauman AE, Booth ML, Ainsworth BE, Pratt M, Ekelund U, Yngve A, Sallis JF, Oja P: International physical activity questionnaire: 12-country reliability and validity. Med Sci Sports Exerc 2003, 35(8):1381-1395.

11. Parks EJ, Skokan LE, Timlin MT, Dingfelder CS: Dietary sugars stimulate fatty acid synthesis in adults. J Nutr 2008, 138(6):1039-1046.

12. Hudgins $L C$, Parker TS, Levine DM, Hellerstein MK: A dual sugar challenge test for lipogenic sensitivity to dietary fructose. J Clin Endocrinol Metab 2011, 96(3):861-868.

13. Nuttall FQ, Khan MA, Gannon MC: Peripheral glucose appearance rate following fructose ingestion in normal subjects. Metab Clin Exp 2000, 49(12):1565-1571.

14. Chong MF, Fielding BA, Frayn KN: Mechanisms for the acute effect of fructose on postprandial lipemia. Am J Clin Nutr 2007, 85(6):1511-1520.

15. Gannon MC, Nuttall FQ, Grant CT, Ercan-Fang S, Ercan-Fang N: Stimulation of insulin secretion by fructose ingested with protein in people with untreated type 2 diabetes. Diabetes Care 1998, 21(1):16-22.

16. Jeppesen J, Chen YD, Zhou MY, Wang T, Reaven GM: Effect of variations in oral fat and carbohydrate load on postprandial lipemia. Am J Clin Nutr 1995, 62(6):1201-1205.

17. Abraha A, Humphreys SM, Clark ML, Matthews DR, Frayn KN: Acute effect of fructose on postprandial lipaemia in diabetic and non-diabetic subjects. Br J Nutr 1998, 80(2):169-175.

18. Cohen JC, Schall R: Reassessing the effects of simple carbohydrates on the serum triglyceride responses to fat meals. Am J Clin Nutr 1988, 48(4):1031-1034.

19. Bohannon NV, Karam JH, Forsham PH: Endocrine responses to sugar ingestion in man. Advantages of fructose over sucrose and glucose. J Am Diet Assoc 1980, 76(6):555-560

20. Bantle JP, Raatz SK, Thomas W, Georgopoulos A: Effects of dietary fructose on plasma lipids in healthy subjects. Am J Clin Nutr 2000, 72(5):1128-1134.

21. Bossetti BM, Kocher LM, Moranz JF, Falko JM: The effects of physiologic amounts of simple sugars on lipoprotein, glucose, and insulin levels in normal subjects. Diabetes Care 1984, 7(4):309-312.

22. Hallfrisch J, Reiser S, Prather ES: Blood lipid distribution of hyperinsulinemic men consuming three levels of fructose. Am J Clin Nutr 1983, 37(5):740-748

23. Crapo PA, Kolterman OG: The metabolic effects of 2-week fructose feeding in normal subjects. Am J Clin Nutr 1984, 39(4):525-534. 
24. Sunehag AL, Toffolo G, Campioni M, Bier DM, Haymond MW: Short-term high dietary fructose intake had no effects on insulin sensitivity and secretion or glucose and lipid metabolism in healthy, obese adolescents. J Pediatr Endocrinol Metab 2008, 21(3):225-235.

25. Singleton MJ, Heiser C, Jamesen K, Mattes RD: Sweetener augmentation of serum triacylglycerol during a fat challenge test in humans. J Am Coll Nutr 1999, 18(2):179-185.

26. Livesey $G$, Taylor R: Fructose consumption and consequences for glycation, plasma triacylglycerol, and body weight: meta-analyses and meta-regression models of intervention studies. Am J Clin Nutr 2008, 88(5):1419-1437.

27. Jeppesen J, Chen, YI, Zhou MY, Schaaf P, Coulston A, Reaven GM: Postprandial triglyceride and retinyl ester responses to oral fat: effects of fructose. Am J Clin Nutr 1995, 61(4):787-791.

28. Rutledge AC, Adeli K: Fructose and the metabolic syndrome: pathophysiology and molecular mechanisms. Nutr Rev 2007, 65 (6 Pt 2):S13-S23.

29. Cirillo P, Gersch MS, Mu W, Scherer PM, Kim KM, Gesualdo L, Henderson GN, Johnson RJ, Sautin YY: Ketohexokinase-dependent metabolism of fructose induces proinflammatory mediators in proximal tubular cells. J Am Soc Nephrol 2009, 20(3):545-553.

30. Delbosc S, Paizanis E, Magous R, Araiz C, Dimo T, Cristol JP, Cros G, Azay J: Involvement of oxidative stress and NADPH oxidase activation in the development of cardiovascular complications in a model of insulin resistance, the fructose-fed rat. Atherosclerosis 2005, 179(1):43-49.

doi:10.1186/1476-511X-13-195

Cite this article as: Jameel et al:: Acute effects of feeding fructose, glucose and sucrose on blood lipid levels and systemic inflammation. Lipids in Health and Disease 2014 13:195.

\section{Submit your next manuscript to BioMed Central and take full advantage of:}

- Convenient online submission

- Thorough peer review

- No space constraints or color figure charges

- Immediate publication on acceptance

- Inclusion in PubMed, CAS, Scopus and Google Scholar

- Research which is freely available for redistribution 